\title{
ON-CHIP SYNTHESIS OF SILICA NANOPARTICLE ASSEMBLIES WITH CONTROLLED SHAPE AND SIZE
}

\author{
J.B. Wacker, V.K. Parashar, and M.A.M. Gijs \\ Laboratory of Microsystems, Ecole Polytechnique Fédérale de Lausanne (EPFL), Lausanne, Switzerland
}

\begin{abstract}
We describe the assembly of silica nanoparticles into superstructures with controlled shape and size. The individual nanoparticles are chemically linked to each other via silica bridges, thus providing high mechanical stability to the assembly. Key to control the size and shape of the assemblies is the confinement of the nanoparticles in highly monodisperse microdroplets that are produced in a microfluidic chip. Exploiting the high surface-to-volume ratio of the assemblies, we show an enzymatic reaction on them. To control the position of the assemblies in a microfluidic environment, we embed superparamagnetic nanoparticles into them, which allows us to move the assemblies with a simple hand magnet.
\end{abstract}

\section{KEYWORDS}

Assembly, nanoparticles, silica, microfluidic droplets

\section{INTRODUCTION}

Three-dimensional superstructures of nanoparticles have a large effective surface [1,2], which is key for their advantageous chemical properties $[3,4]$. They are, for example, excellent supports for catalysts. Assembly of silica nanoparticles into solid objects of controlled shape is still lacking, despite the high tolerance of silica to solvents, combined with an excellent mechanical and thermal stability [5]. Nanoparticle assembly on microfluidic chips is a promising technique due to its facile process control. A way to tune the morphology of the assembly is to confine the nanoparticles in monodisperse nanolitre colloidal droplets. While top-down micro-emulsification produces droplets with a big variety of sizes [6], the diameter of individual droplets can be extremely well controlled in a microfluidic circuit by pinching-off the colloid flow with secondary immiscible flows $[7,8]$. Few research articles show the synthesis of inorganic microparticles from sol precursors in droplets $[9,10]$. Others showed the assembly of inorganic nanoparticles at the surface of microdroplets to create hollow capsules that can, for instance, comprise and release drugs [11-14].

While spherical assemblies are prepared in a natural way by simply evaporating the liquid phase from droplets that contain the nanoparticles, the synthesis of non-spherical microstructures requires more elaborate techniques, for instance controlling the coalescence between spherical droplets [15] or assembling nanoparticles in non-spherical double droplets [16].

In previous studies, cohesion between the nanoparticles in the assembly was assured either by van der Waals forces [2, 17], hydrophobic interactions between functionalized nanoparticles [14], adsorbing polyelectrolytes on the assembly [13], cross-linking the nanoparticles with reactive molecules [18] or embedding the nanoparticles in an organic photopolymerizable matrix [19]. However, these procedures lead to assemblies with a low chemical and mechanical stability. While fusing organic nanoparticles via sintering into consolidated assemblies was demonstrated, for inorganic assemblies, such approach would involve a high-temperature process $\left(T>1000^{\circ} \mathrm{C}\right)$, incompatible with organic or magnetic moieties within the assembly.

In our approach, we functionalized the surface of silica nanoparticles with a silanol-containing gel-layer that solidifies at moderate temperature $\left(460^{\circ} \mathrm{C}\right)$ and thereby physically links the individual nanoparticles in an assembly, leading to mechanically and chemically stable assemblies of silica nanoparticles.

\section{MATERIALS AND METHODS \\ Chip Fabrication}

If not indicated differently, chemicals were purchased from Sigma Aldrich, Switzerland.

Glass chips were produced using standard photolithography and etching technique. In brief, chromium and gold layers $(20 \mathrm{~nm}$ and $100 \mathrm{~nm}$, respectively) were evaporated on float glass wafers with a Leybold optics LAB $600 \mathrm{H}$ evaporator. Subsequently, a $1.5 \mu \mathrm{m}$ thin layer of photoresist (AZ1512, AZ electronic materials, Germany) was spun onto the gold layer. The channels were patterned into the photoresist by exposing the parts to be removed to UV light (17 mJ intensity), followed by development with AZ726MIF. Gold was etched using a mixture of $\mathrm{KI}$ and $\mathrm{I}_{2}(25 \mathrm{~g} / \mathrm{L}$ and $12 \mathrm{~g} / \mathrm{L})$, and chromium was removed with chromium etchant CR-7 (from Cyantek, USA). Channels with a depth of $40 \mu \mathrm{m}$ were subsequently trenched into glass during 8 minutes in a $12 \% \mathrm{HF}$ bath at $35^{\circ} \mathrm{C}$. After confirming the channel depth with a capacitive profilometer (Tencor-Alphastep 500), photoresist was removed with Shipley's Microposit Remover $1165\left(2 \times 15\right.$ minutes at $\left.70^{\circ} \mathrm{C}\right)$. Gold and chromium were then completely etched away using the previously mentioned chemicals. Afterwards, the wafers were cut into chips with a dicing saw (Disco DAD 321). Holes for the inlets and the outlet were formed with a powder-blasting machine. The glass piece containing the channels and the glass chip with the holes were then thermally bonded at $645^{\circ} \mathrm{C}$ during 4 hours. To make the channels hydrophobic, we flooded them with trichloro(1H,1H,2H,2H-perfluorooctyl)silane for 30 minutes. Finally, connectors (NanoPorts from IDEX), were glued on the inlets, and to clamp a capillary over the outlet, a piece of cured polydimethylsiloxane (PDMS, from Dow Corning) containing a hole was aligned over 
the outlet and plasma-bonded to the chip.

\section{Nanoparticle Synthesis}

Nanoparticles were synthesized from tetraethyl orthosilicate (TEOS) and a hydrolyzing mixture (HM) containing $40 \% \mathrm{v} / \mathrm{v}$ de-ionized (DI) water, $12 \% \mathrm{v} / \mathrm{v}$ ethanol and $48 \% \mathrm{v} / \mathrm{v}$ aqueous ammonia. $0.5 \mathrm{~mL}$ TEOS and $1 \mathrm{~mL} \mathrm{HM}$ were repeatedly injected into a glass vial after $5,8,10,12,14, \ldots 20$ minutes and mixed under stirring at $300 \mathrm{rpm}$. Two minutes after the last injection, the vial was put in a temperature-stabilized bath at $40^{\circ} \mathrm{C}$, and stirring was increased to $700 \mathrm{rpm}$ for 30 minutes. We then centrifuged and washed the nanoparticles. Supernatant water was removed by heating the nanoparticles to $60^{\circ} \mathrm{C}$ overnight. After pounding nanoparticle agglomerates to a fine powder, we calcined them at $460^{\circ} \mathrm{C}$ overnight. To form a gel-layer containing silanol on the nanoparticles, we autoclaved them in DI water for 10 minutes at $120^{\circ} \mathrm{C}$ under indigenously built pressure.

\section{Droplet-Based Assembly}

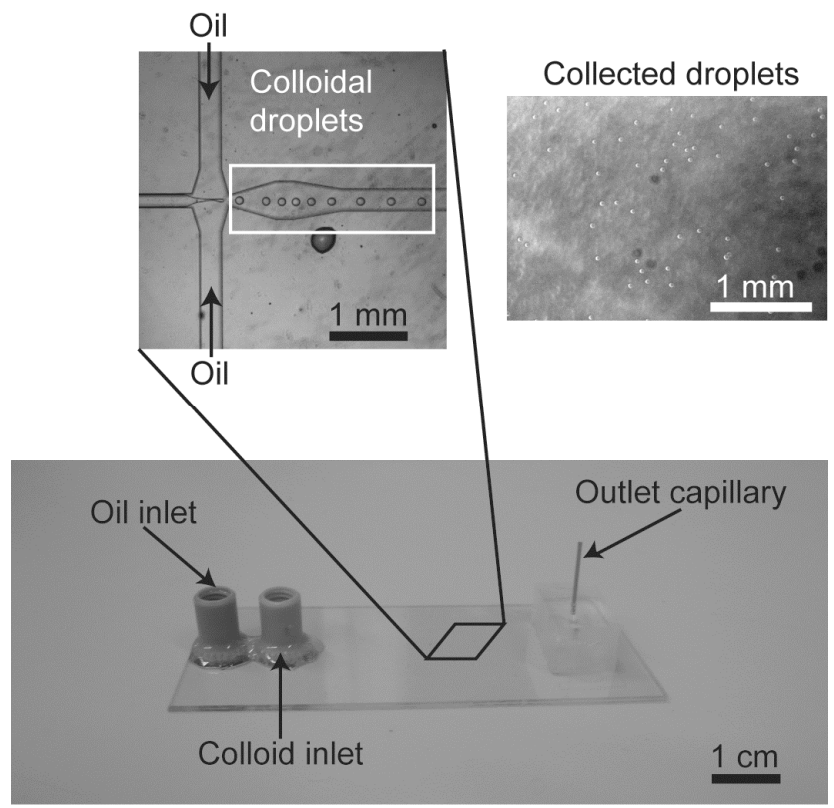

Figure 1 : Experimental setup. By pinching-off an aqueous silica colloid stream with oleic acid in a microfluidic chip, colloidal droplets are formed and subsequently collected in a PDMS container.

Silica nanoparticles with a gel-layer were dispersed in DI water at weight concentrations between $0.01 \%$ and $5 \%$. Droplets loaded with nanoparticles were formed by injecting this colloid in the central channel of the chip and oleic acid in the two side channels (see Fig. 1). Typically, the flow rates were $0.001-0.005 \mu \mathrm{L} / \mathrm{sec}$ for the colloid and $0.05-0.075 \mu \mathrm{L} / \mathrm{sec}$ for the oil. The droplets were then collected in a PDMS container at the outlet capillary. To avoid coalescence, the container was slowly shifted, so to have a distance of at least $100 \mu \mathrm{m}$ between the individual droplets. To make pillar-like assemblies, the droplets were rolled over the bottom of the container at a frequency of $0.5 \mathrm{~Hz}$. Magnetic assemblies were produced by doping the silica colloid with $0.1 \% \mathrm{w} / \mathrm{w}$ iron oxide nanoparticles (7 $\mathrm{nm}$ in diameter) [20]. To evaporate the water from the droplets, the PDMS container with the oil and the colloidal droplets was heated to $160^{\circ} \mathrm{C}$ for 2 hours. After washing with water, the assemblies were studied under a scanning electron microscope (SEM, XLF-30, Philips) or (for fluorescence experiments) under an upright optical microscope (Imager A1.m, Zeiss, Germany).

\section{Catalysis Experiments}

Catalytic experiments with horseradish peroxidase (HRP, TMA kit \#42 from Invitrogen, Switzerland) were conducted in a micro flow chamber that consisted of a microscope cover slip which was glued on top of a microscope slide using double sticky tape (from $3 \mathrm{M}$, Switzerland). The walls of this micro flow chamber were passivated with $0.1 \mathrm{mg} / \mathrm{mL}$ poly-L-lysine $(20 \mathrm{kDa})$ grafted with polyethylene glycol $(2 \mathrm{kDa})$ (PLL-g-PEG, from Susos, Switzerland) in blocking solution (BS). Nanoparticle assemblies were then inserted into the flow chamber, held in place with a hand magnet and covered with biotinylated PLL-g-PEG $(0.1 \mathrm{mg} / \mathrm{mL}$ in BS $)$. Subsequently, streptavidin-conjugated HRP was allowed to bond to the biotin for 1 hour. After washing with BS, dye-labeled tyramide in $0.0015 \% \mathrm{v} / \mathrm{v} \mathrm{H}_{2} \mathrm{O}_{2}$ catalytically bound to the assemblies. After 5 minutes, unbound tyramide was washed away with BS, and fluorescence was observed under an optical microscope.

\section{RESULTS AND DISCUSSION Assemblies of Different Sizes and Shapes}

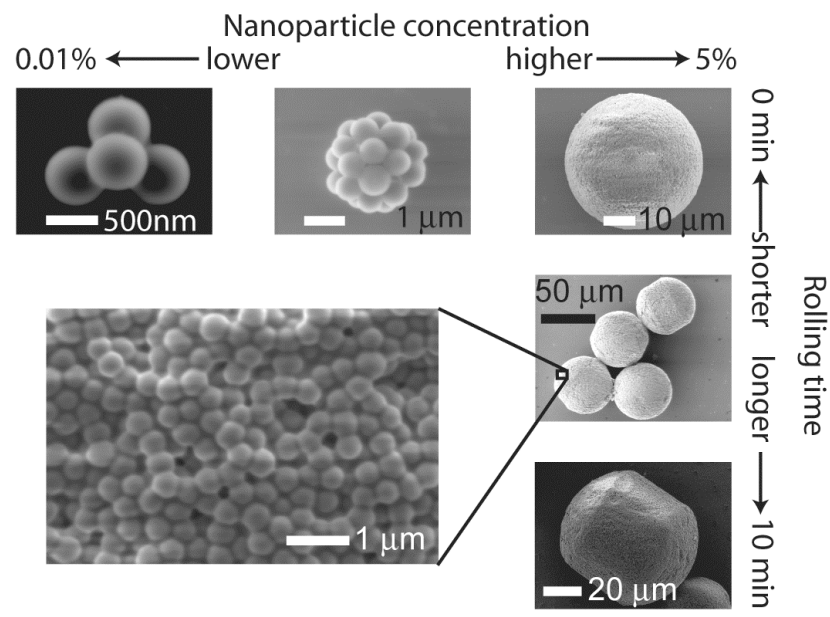

Figure 2 : SEM micrographs of nanoparticle assemblies formed in microdroplets. By adjusting the nanoparticle concentration and the droplet rolling time, the size and the shape of the assemblies can be controlled. The lower-left micrograph is a zoom on the individual nanoparticles held together via silica bridges that were formed by a solidified silica gel layer on the particles. 
Control over the size of the assemblies can be achieved by reducing the size of the droplets or by lowering the nanoparticle concentration per droplet. We opted for the latter tuning parameter, since the diameter of droplets is stable over a wide range of flow velocities when using oleic acid and water as continuous and dispersed phases, respectively [21]. At a low nanoparticle concentration $(0.01 \% \mathrm{w} / \mathrm{w})$, small agglomerates of 2 to 10 nanoparticles were obtained (see Fig. 2). Small spherical assemblies formed at a medium concentration of $0.1 \% \mathrm{w} / \mathrm{w}$, and perfectly spherical assemblies resulted from experiments with high nanoparticle concentration $(5 \% \mathrm{w} / \mathrm{w})$. As can be seen in Fig. 1, the droplets in which the nanoparticles were assembled, had a low size distribution. This is reflected by the high monodispersity of the spherical superstructures (variance $/$ mean $=2 \%$ ).

In addition to spherical assemblies, we also formed non-spherical, pillar-like assemblies (see Fig. 2). These are created by rolling the colloidal droplets over a hydrophobic surface. The aspect ratio of the pillar-like assemblies was controlled by adjusting the time during which the droplets were moved; longer rolling times correspond to more elongated assemblies.

The lower-left photograph in Fig. 2 is a high magnification SEM micrograph, showing that individual nanoparticles are linked to each other via silica bridges. These bridges are found in all presented assemblies. They form from the silica gel layer on the nanoparticles that solidifies when heating the assemblies to $460^{\circ} \mathrm{C}$. We assessed the mechanical stability of the assemblies in compression experiments and found a breaking pressure of $393 \mathrm{kPa}$ and a modulus of elasticity of $104 \mathrm{MPa}$. Assemblies without silica bridges simply collapse under their proper weight, thus not allowing mechanical testing.

\section{Magnetic Assemblies}

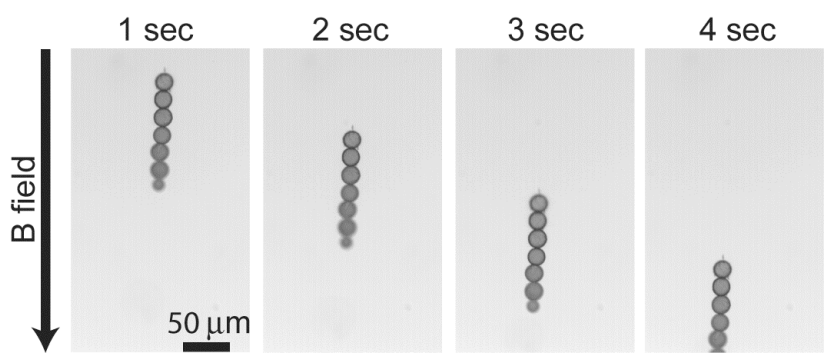

Figure 3 : Movement of a chain of magnetic nanoparticle assemblies aligned in a magnetic field.

Magnetic assemblies were formed by embedding superparamagnetic iron oxide nanoparticles into the assembly. This allows manipulating the assemblies without physical contact force. Fig. 3 is a time-lapse sequence of spherical magnetic silica nanoparticle assemblies lining up in a B-field and moving through water. The magnetophoretic mobility of a magnetic spherical object of radius $R$ describes how magnetically manipulable the object is in a viscous medium and is given by $\xi=\left(2 R^{2} \Delta \chi\right) /(9 \eta)$, where $\Delta \chi$ is the difference in magnetic susceptibility between the magnetic assembly and the surrounding liquid medium (e.g. water), and $\eta$ the viscosity of water [22]. Since $\xi$ scales with $R^{2}$, microscopic assemblies allow much easier magnetic manipulation than individual magnetic nanoparticles.

\section{Use as a Catalytic Support}

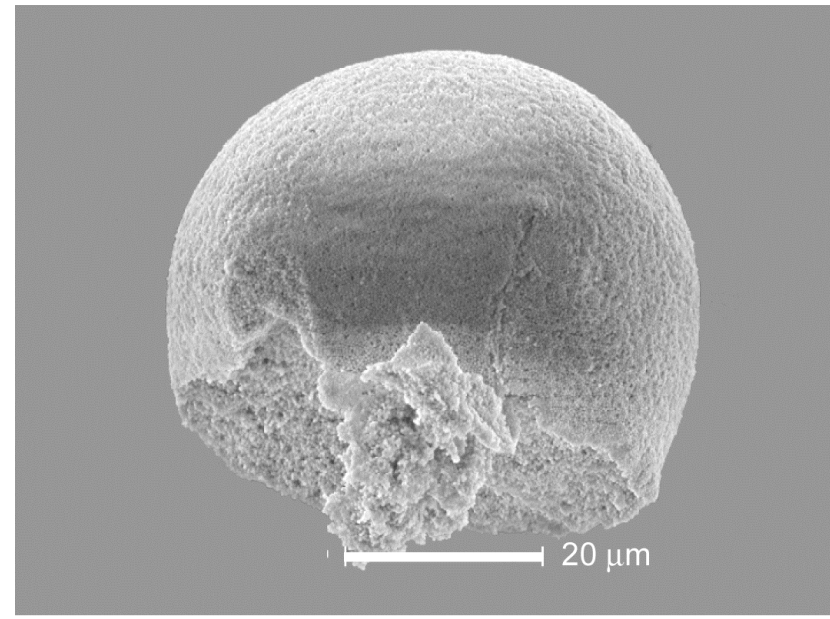

Figure 4 : A broken nanoparticle assembly, showing the solid, porous interior.

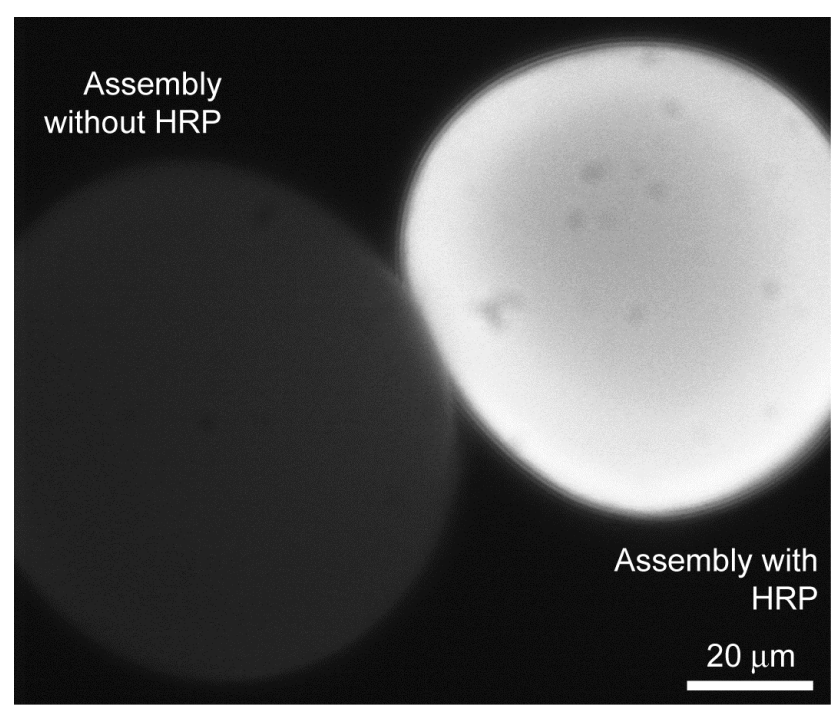

Figure 5 : The assemblies can serve as catalytic supports. Only assemblies decorated with HRP develop catalytically induced fluorescence.

As can be seen in Fig. 4, the presented assemblies are porous and solid. They therefore have a surface which is much bigger than the surface of a monolithic sphere of the same radius. A huge surface-to-volume ratio is a prerequisite for catalytic supports. We have functionalized the assemblies with the enzyme HRP and observed catalytic precipitation of a fluorescent dye not only on the outer surface of the assembly, but also in the interior, porous structure, confirming the catalytic 
activity of HRP on the assembly. As a negative control, an experiment with an assembly lacking HRP was run in parallel, showing no fluorescence (see Fig. 5).

\section{CONCLUSION}

In conclusion, we took advantage of the high monodispersity of on-chip generated microdroplets to produce nanoparticle assemblies with narrow size distribution. By rolling the droplets over a hydrophobic surface, we created pillar-like assemblies.

We doped the assemblies with superparamagnetic particles and could easily control their position in a micro flow cell. Utilizing the high surface-to-volume ratio of the porous superstructures, we used them as a support for a catalytic precipitation reaction of a fluorescent dye. We speculate that many other catalytic reactions will be realized on these nanoparticle assemblies. Since silica is a chemically very stable material and due to the high mechanical stability of the assemblies, washing off reaction products and catalysts from previous experiments under harsh conditions will be feasible.

\section{REFERENCES}

[1] O.D. Velev, A.M. Lenhoff, E.W. Kaler, "A Class of Microstructured Particles Through Colloidal Crystallization", Science, 287, pp.2240-2243, 2000.

[2] A. Dinsmore, M. Hsu, M. Nikolaides, M. Marquez, A. Bausch, D. Weitz, "Colloidosomes: Selectively permeable capsules composed of colloidal particles", Science, 298, pp.1006-1009, 2002.

[3] D.R. Rolison, "Catalytic nanoarchitectures - The importance of nothing and the unimportance of periodicity", Science, 299, pp.1698-1701, 2003.

[4] K. Burkert, T. Neumann, J.J. Wang, U. Jonas, W. Knoll, H. Ottleben, "Automated preparation method for colloidal crystal arrays of monodisperse and binary colloid mixtures by contact printing with a pintool plotter", Langmuir, 23, pp.3478-3484, 2007.

[5] R.G. Newton, A. Paul, "A New Approach to Predicting the Durability of Glasses from Their Chemical Compositions", Glass Technology, 21, pp.307-309, 1980.

[6] S.Y. Teh, R. Lin, L.H. Hung, A.P. Lee, "Droplet microfluidics", Lab Chip, 8, pp.198-220, 2008.

[7] G.F. Christopher, S.L. Anna, "Microfluidic methods for generating continuous droplet streams", J. Phys. D-Appl. Phys., 40, pp.R319-R336, 2007.

[8] Q.Y. Xu, M. Nakajima, "The generation of highly monodisperse droplets through the breakup of hydrodynamically focused microthread in a microfluidic device", Appl. Phys. Lett., 85, pp.3726-3728, 2004.

[9] I. Lee, Y. Yoo, Z. Cheng, H.-K. Jeong, "Generation of Monodisperse Mesoporous Silica Microspheres with Controllable Size and Surface Morphology in a Microfluidic Device", Advanced Functional Materials, 18, pp.4014-4021, 2008.

[10] N.J. Carroll, S.B. Rathod, E. Derbins, S. Mendez, D.A. Weitz, D.N. Petsev, "Droplet-Based
Microfluidics for Emulsion and Solvent Evaporation Synthesis of Monodisperse Mesoporous Silica Microspheres", Langmuir, 24, pp.658-661, 2008.

[11] A. Boker, J. He, T. Emrick, T. Russell, "Self-assembly of nanoparticles at interfaces", Soft matter, 3, pp.1231-1248, 2007.

[12] D. Lee, D.A. Weitz, "Double emulsion-templated nanoparticle colloidosomes with selective permeability", Adv. Mater., 20, pp.3498-3503, 2008.

[13] I. Akartuna, E. Tervoort, A. Studart, L. Gauckler, "General Route for the Assembly of Functional Inorganic Capsules", Langmuir, 25, pp.12419-12424, 2009.

[14] M.H. Lee, V. Prasad, D. Lee, "Microfluidic Fabrication of Stable Nanoparticle-Shelled Bubbles", Langmuir, 26, pp.2227-2230, 2009.

[15] A.R. Studart, H.C. Shum, D.A. Weitz, "Arrested Coalescence of Particle-coated Droplets into Nonspherical Supracolloidal Structures ", The Journal of Physical Chemistry B, 113, pp.3914-3919, 2009.

[16] D. Lee, D.A. Weitz, "Nonspherical Colloidosomes with Multiple Compartments from Double Emulsions", Small, 5, pp.1932-1935, 2009.

[17] M.F. Hsu, M.G. Nikolaides, A.D. Dinsmore, A.R. Bausch, V.D. Gordon, X. Chen, J.W. Hutchinson, D.A. Weitz, M. Marquez, "Self-assembled Shells Composed of Colloidal Particles: Fabrication and Characterization", Langmuir, 21, pp.2963-2970, 2005.

[18] Y. Lin, H. Skaff, A. Böker, A.D. Dinsmore, T. Emrick, T.P. Russell, "Ultrathin Cross-Linked Nanoparticle Membranes", Journal of the American Chemical Society, 125, pp.12690-12691, 2003.

[19] S. Kim, J. Shim, J. Lim, S. Lee, S. Yang, "Microfluidic fabrication of microparticles with structural complexity using photocurable emulsion droplets", New journal of physics, 11, pp.075014, 2009.

[20] M. Chastellain, A. Petri, H. Hofmann, "Particle size investigations of a multistep synthesis of PVA coated superparamagnetic nanoparticles", Journal of Colloid and Interface Science, 278, pp.353-360, 2004.

[21] J. Wacker, V.K. Parashar, M.A.M. Gijs, "Influence of Oil Type and Viscosity on Droplet Size in a Flow Focusing Microfluidic Device", Proceedings of the Eurosensors Xxiii Conference, Lausanne, September 6-9, 2009, pp.1083-1086.

[22] M.A.M. Gijs, F. Lacharme, U. Lehmann, "Microfluidic Applications of Magnetic Particles for Biological Analysis and Catalysis", Chem. Rev., 110, pp.1518-1563, 2010.

\section{CONTACT}

* J. Wacker, tel: 41-21-693-6584; josias.wacker@epfl.ch 